Methods A comprehensive search of published studies was carried out in six electronic databases followed by a manual search of studies from references of selected papers. Data were extracted using a template. The results were synthesised, and a meta-analysis based on a random-effects model was conducted. Subgroup and sensitivity analyses were undertaken to explore sources of heterogeneity.

Results Of 30,273 citations, 14 studies with a total of 97,030 study participants were identified. The pooled CHTC uptake was 31.48\% (95\%CI: 23.55-40.00) with significant heterogeneity between studies $\left(\mathrm{I}^{2}=99.98 \%, \mathrm{p}<0.001\right)$. The Egger's and Begg's tests showed there was no evidence of publication bias $(p=0.08)$. However, the sensitivity analysis showed that two studies highly influenced the overall estimate. After omitting these two studies, the pooled estimate for CHTC uptake was $24.05 \%$ (95\%CI: $\left.16.65,32.34, \mathrm{I}^{2}=99.86 \%, \mathrm{p}<0.001\right)$. The sub-group analysis indicated the pooled CHTC uptake was higher among pregnant women and their partners $(\mathrm{OR}=1.66,95 \% \mathrm{CI}: 1.58,1.84)$ compared with heterosexual couples in general. Similarly, the uptake was higher when one person in the dyad first tested individually without the knowledge of their partner, and then suggested to their partner that they take CHTC together, compared to an approach of undertaking CHTC together as the first testing option for both people (OR=3.16, 95\%CI: 2.69, 3.72).

Conclusion The findings confirmed that more than three-quarters of study participants who were in ongoing heterosexual relationships chose not to, or were unable to, undertake CHTC. These findings suggest people are cautious of what could amount to harmful risks when couples test together, particularly if their HIV sero-status is shown to be discordant. Further studies are required to explore how couples intend to use HIV testing services including CHTC.

Disclosure No significant relationships.

\section{P008 DIAGNOSTIC ACCURACY OF XPERT MTB/RIF IN DETECTING PULMONARY TUBERCULOSIS AMONG PEOPLE LIVING WITH HIV IN WESTERN NIGERIA}

Saheed Usman*, Prosper Okonkwo, Oluwatoyin Jolayemi, Jay Osi-Samuels, Patrick Akande, Babatunde Ladi-Akinyemi, Oluremi Olaitan, Femi Owolagba, Matthias Alagi, Eke Ofuche. APIN Public Health Intiatives, Abuja, Nigeria

\subsection{6/sextrans-2019-sti.220}

Background Tuberculosis is a leading killer among people living with human immunodeficiency virus (HIV). HIV-infected individuals with latent $\mathrm{TB}$ are approximately 20-30 times more likely to develop TB disease, at a rate of $8-10 \%$ per year, with the disease estimated to cause approximately 9 million cases annually and 1.5 million deaths. Hypothesis tested was site of infection effect on tuberculosis on tuberculosis treatment outcome. This study determined the diagnostic validity and reliability of Xpert MTB/RIF in identifying the presence of Pulmonary Tuberculosis (PTB) among HIV patients in South Western Nigeria.

Methods This study was a prospective analytical study among HIV patients between ages $15-60$ years who are infected with HIV seen from January 2015 - June 2017. Patients with signs and symptoms of Pulmonary Tuberculosis (PTB) were enrolled and submitted sputum for Acid Fast Bacilli (AFB) smear and Xpert MTB/RIF. This was processed following protocol for pulmonary samples for Xpert MTB/Rif. All samples were processed for AFB smear and Xpert MTB/RIF as part of the procedure for PTB diagnosis.

Results A total of 300 patients were enrolled in the study. The mean age \pm SD is $37.11 \pm 15.27$ years. One hundred and thirty five $(45.0 \%)$ of them are males while one hundred and sixty five $(55.0 \%)$ are females. Xpert MTB/RIF has a sensitivity of $93.0 \%$ and specificity of $98.5 \%$. The main factor associated with tuberculosis treatment outcome was the site of infection $\left(\chi^{2}=19.01\right.$, df $\left.=1, \mathrm{p}=0.001\right)$ as $233(77.7 \%)$ of the patients were declared cured after six month treatment course.

Conclusion Use of Xpert MTB/RIF as a screening tool has a great performance for rapid diagnosis of Mycobacterium tuberculosis might effectively reduce the risk of multi-drug resistant tuberculosis (MDR-TB) in HIV care and treatment settings and improve the prognosis of affected patients.

Disclosure No significant relationships.

\section{P012 A PROCESS EVALUATION OF AN INCENTIVIZED HOME- BASED INTERVENTION TO TEST AND START (HITS) IN RURAL KWAZULU-NATAL, SOUTH AFRICA}

${ }^{1}$ Oluwafemi Adeagbo* ${ }^{1}$ 'Frank Tanser, ${ }^{1}$ Velaphi Gumede, ${ }^{2}$ Thulile Mathenjwa, ${ }^{3}$ Philippa Matthews, ${ }^{4}$ Sally Wyke, ${ }^{5}$ Till Bärnighausen, ${ }^{2}$ Deenan Pillay, ${ }^{6}$ Nuala Mcgrath, ${ }^{7}$ Ann Blandford, ${ }^{8}$ Janet Seeley, ${ }^{9}$ Maryam Shahmanesh. ${ }^{7}$ Africa Health Research Institute, Social Science Research and Ethics, Mtubatuba, South Africa; ${ }^{2}$ Africa Health Research Institute, Mtubatuba, South Africa; ${ }^{3}$ Africa Health Research Institute, Clinical Research, Mtubatuba, South Africa; ${ }^{4}$ University of Glasgow, College of Social Sciences, Glasgow, UKi ${ }^{5}$ Heidelberg University, Heidelberg, Germany; ${ }^{6}$ University of Southampton, Southampton, UK; ${ }^{7}$ University College London, Institute of Digital Health, London, UK; ${ }^{8}$ London School of Hygiene and Tropical Medicine, London, UK; ${ }^{9}$ University College London, London, UK

10.1136/sextrans-2019-sti.221

Background Despite freely available HIV testing and treatment, many men do not access HIV treatment and care in South Africa. We conducted home-based intervention to test and start (HITS) - a factorial design randomised controlled trial (ClinicalTrials.gov \#NCT03757104). HITS is designed to assess the effectiveness of two financial micro-incentives (R50 [\$3] food vouchers) for home-based HIV testing and, following a HIV-positive test, to link to HIV care; and/or a maletargeted counselling application to support home-based testing (EPIC-HIV1) and to support men linking to care (EPICHIV2). The research was conducted in an HIV-hyperendemic setting in rural KwaZulu-Natal.

Methods We conducted a process evaluation to understand the impact of HITS intervention on the decision of men to test for HIV and/or engage in HIV care. Thirty men (16-73 years) were purposively selected in the three intervention arms (ten per arm) and interviewed between August and December 2018. Emerging themes were thematically analysed following an interpretivist approach. 
Results Although participants welcomed the male-centred intervention, some felt that the voucher amount was too small. Overall, many participants described the voucher, EPIC-HIV, and the convenience and privacy of home-based testing as 'catalysts' to test or link to care irrespective of their reported intrinsic motivations to know their status or concerns around HIV related sexual risk behaviours. One-third of the interviewees were first-time testers. Despite the incentives, two out of the five men who tested positive reported that they have not linked to care because they feared stigmatisation at local clinics.

Conclusion Generally, the HITS intervention influenced men's motivation to test and access care, but some respondents felt the incentive was insufficient to overcome some barriers of accessing HIV care at fixed clinics. To achieve the 90-90-90 targets among men in our setting, provision of decentralised, non-judgmental and convenient incentivised HIV care services could increase uptake of HIV testing and treatment.

Disclosure No significant relationships.

\section{P013 SCREENING PRACTICES RELATED TO INCONCLUSIVE NEISSERIA GONORRHOEAE AND CHLAMYDIA TRACHOMATIS NUCLEIC ACID AMPLIFICATION TESTING}

${ }^{1}$ Cherie Blair*, ${ }^{1}$ Omai Garner, ${ }^{2}$ Bettina Pedone, ${ }^{2}$ Sam Elias, ${ }^{1}$ Raphael Landovitz. ${ }^{1}$ University of California, Los Angeles, Department of Medicine, Division of Infectious Diseases, Los Angeles, USA; ' University of California, Los Angeles, Arthur Ashe Student Health and Wellness Center, Los Angeles, USA

\subsection{6/sextrans-2019-sti.222}

Background Given rising incidence of Neisseria gonorrhoeae and Chlamydia trachomatis (GC/CT), development of efficacious screening strategies is critical. While guidelines recommend nucleic acid amplification testing (NAAT) for GC/CT screening, a small proportion of NAAT results are inconclusive - resulting in delays in diagnosis and treatment. Our study seeks to determine rates as well as provider- and patientrelated factors associated with inconclusive NAAT results with the goal of developing improved screening practices.

Methods This is a cross-sectional, case-control study of individuals with inconclusive GC/CT NAATs at a single institution (University of California, Los Angeles) from 3/2016-6/2018. Clinical charts were abstracted for age, gender, HIV status, PrEP use, STI screening (GC/CT and syphilis), anatomic site of sample collection (urogenital, pharyngeal, or rectal), and whether repeat testing occurred within 6 months following an inconclusive result. Cases were defined as a specimen that resulted in inconclusive GC and/or CT NAAT. Controls were specimens randomly selected from all non-inconclusive samples that underwent GC/CT NAAT during the study period and were matched with inconclusive specimens by type (rectal, urine, or genital). Patient-level characteristics associated with inconclusive GC/CT testing were analyzed with Chi-square and logistic regression.

Results During the study period, 6.1\% (852/14,048) rectal, $0.3 \% \quad(313 / 91,092)$ genital, and $0.01 \% \quad(155 / 137,783)$ urine samples were inconclusive for one or both of GC and CT; no pharyngeal samples yielded inconclusive results. Among patients with inconclusive GC/CT NAAT, 64.6\% (441/683) received repeat testing within six months, of which $6.4 \%$ were positive, $82.8 \%(365 / 441)$ negative, $10.7 \%$ (47/441) inconclusive, and $0.2 \%(1 / 441)$ indeterminate. While diabetes was associated with inconclusive urogenital GC/CT NAAT,
HIV status, PrEP use, and positive STI screening were not associated with inconclusive results.

Conclusion Despite having a clinical indication to receive GC/ CT screening, fewer than two-thirds of inconclusive results were repeated, potentially missing an opportunity to interrupt the infection cycle.

Disclosure No significant relationships.

\section{P014 VALIDATING A CLINICAL PREDICTION RULE FOR CHLAMYDIA AND GONORRHEA INFECTION AMONG ONLINE TESTERS IN BRITISH COLUMBIA, CANADA}

\begin{abstract}
${ }^{1}$ Aidan Ablona*, ${ }^{2}$ Titilola Falasinnu, ${ }^{1}$ Michael Irvine, 'Devon Haag, ${ }^{1} H$ Hiu-Ju Chang, ${ }^{3}$ Sharmistha Mishra, ${ }^{3}$ Ann Burchell, ${ }^{4}$ Christopher Fairley, ${ }^{5}$ Nathan Lachowsky, ${ }^{6}$ Paul Flowers, ${ }^{6}$ Claudia Estcourt, ${ }^{7}$ Michelle Murti, ${ }^{1}$ Oralia Gomez-Ramirez, ${ }^{8}$ Mel Krajden, ${ }^{1}$ Troy Grennan, ${ }^{1}$ Mark Gilbert. ${ }^{1} B C$ Centre for Disease Control, Clinical Prevention Services, Vancouver, Canada; ${ }^{2}$ Stanford University, Department of Health Research and Policy, Stanford, USA; ${ }^{3}$ St Michael's Hospital, Li Ka Shing Knowledge Institute, Toronto, Canada; ${ }^{4}$ Monash University, Central Clinical School, Carlton, Australia; ${ }^{5}$ University of Victoria, School of Public Health and Social Policy, Victoria, Canada; ${ }^{6}$ Glasgow Caledonian University, School of Health and Life Sciences, Glasgow, UK; ${ }^{7}$ Public Health Ontario, Toronto, Canada; ${ }^{8} B C$ Centre for Disease Control, Public Health Laboratory, Vancouver, Canada
\end{abstract}

10.1136/sextrans-2019-sti.223

Background Clinical prediction rules (CPRs) estimate the probability of a health outcome to support decision-making in intervention and service delivery. Previously, a CPR was derived to maximize detection of chlamydia and/or gonorrhea (CT/GC) infections and minimize the number of screening tests offered among asymptomatic women and heterosexual men attending sexually-transmitted infection (STI) clinics in Vancouver, British Columbia, Canada. We assessed the external validity of using this clinic-derived CPR within GetCheckedOnline (GCO), a provincial online STI testing program in British Columbia.

Methods Data used for calculating CPR scores, including age, race/ethnicity, number of sexual partners and previous CT/GC diagnoses, were collected prospectively on GCO from October 2015 to June 2018. Model calibration and discrimination were evaluated using the Hosmer-Lemeshow (H-L) statistic and the area under the receiver operating characteristic curve (AUC), respectively. Sensitivity and proportion of GCO clients screened were calculated at different CPR cut-off scores. In the original derivation population, the CPR had an $\mathrm{AUC}=0.74$, with a cut-off risk score $\geq 6$ identifying $91 \%$ of infections and screening $68 \%$ of testers.

Results Among 2703 GCO CT/GC test episodes, the prevalence of CT/GC infection was $2.1 \%$. The clinic CPR showed reasonable calibration $(\mathrm{H}-\mathrm{L} \quad \mathrm{p}=0.952)$ and discrimination (AUC $=0.64,95 \% \mathrm{CI}:$ 0.57-0.71). Using a CPR cut-off risk score of $\geq 6$, we would have detected $79 \%$ of infections and screened $64 \%$ of testers. Lowering the cut-off risk score to $\geq 4$ would have increased sensitivity to $95 \%$ while screening $85 \%$ of testers.

Conclusion This is the first study validating the use of a clinic-derived CPR within an online setting. Our CPR showed reasonable accuracy and performance when applied to GCO data. Differences in model performance online compared with clinic-based settings highlight important differences in the populations who use online testing. Use of CPRs in online contexts offers unique and novel opportunities for public health and STI testing.

Disclosure No significant relationships. 